\title{
Matching stereoscopic SAR images for radargrammetric applications
}

\author{
Franck Fayard, Stéphane Méric, Eric Pottier \\ SAPHIR team / IETR, Université de Rennes 1 \\ Bat 11D, 263 av du Général Leclerc, CS 74205 \\ 35042 Rennes cedex, France \\ franck.fayard@ens.insa-rennes.fr
}

\begin{abstract}
The aim of this paper is to present our studies about extraction of 3D information from radar images. Several radargrammetric methods allow DEM (Digital Elevation Model) generation from SAR images and we take a special interest to stereoscopic method. The main idea is to match image stereo pairs, to create a disparity map from one image to the other and to compute elevation thanks to the incidences angles.
\end{abstract}

\section{Radargrammetry, stereoscopy, matching images}

\section{INTRODUCTION}

The research activities of the S.A.P.H.I.R (SAR Polarimetry, Holography, Interferometry and Radargrammetry) team are in the field of Synthetic Aperture Radar (SAR) technology, especially multidimensional systems. A special interest is deserved to develop theories on radar polarimetry, interferometry and teledetection. These activities allow the research group to simulate SAR images and to analyze SAR data qualitatively and quantitatively: surface roughness, soil moisture, snow and forest covers, urban areas...

The mastery of this knowledge motivates the team to develop studies on the stereoscopic area. The aim of this paper is to present the realization of a radargrammetric processing. This paper is actually a presentation of improvements of the first works managed in SAPHIR team. This processing makes it possible to generate DEM from stereoscopic SAR images.

Succinctly, we will present different radargrammetric techniques. Then, we will expose our works: the first tests are on synthesized SAR images to validate elementary results as DEM extraction from several scatterers on the radar images [1]. Then, we use different stereoscopic image pairs to make evolve the algorithm in order to work on high-resolution SAR images.

\section{STATE OF THE ART}

\section{A. Different radargrammetric methods}

Radargrammetry is similar to photogrammetry for optical images: radargrammetry is defined as "the technology of extracting geometric information from radar images" [2]. Several radargrammetric methods allow DEM generation from SAR images: clinometry, stereoscopy, interferometry and polarimetry [3]. The choice of a radargrammetric technique depends on the SAR configuration, the means used during the image acquisition and the relief type. For example, stereoscopy and interferometry involve the recording of two (or more) images, while polarimetry requires recording several data channels: HH, VV, HV, VH.

The most popular radargrammetric techniques are the interferometry and stereoscopy. Clinometry remains a marginal technique for DEM generation, applied mainly in difficult situations such as sites without ground references. Polarimetry is limited by the lack of polarimetric data to evaluate the robustness of this emergent technique. Interferometry is a wellknown method [4], which offers the best accuracy for DEM extraction but phase unwrapping and propagation effects can corrupt the differential phase.

\section{B. Stereoscopic method principle}

Stereoscopy consists in recording two images of the same area with different incidence angles. The crucial step is to match the two images in order to identify the homologous points from one image to the other. Once the matching process finished, the differences between images can be measured to establish a disparity map. This disparity map is used in radargrammetry to compute the terrain elevation from the measured parallaxes between the two images. On the assumption that the area is flat, the flight lines are parallel and the height flight is constant, [2] allows using (1) for a slantrange presentation and (2) for a ground-range presentation.

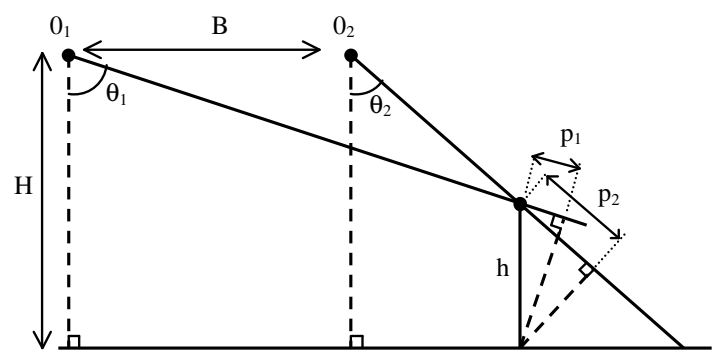

Figure 1. Stereo configuration

$$
\begin{aligned}
& \mathrm{h}=\left(\mathrm{p}_{1}-\mathrm{p}_{2}\right) /\left(\cos \theta_{2} \pm \cos \theta_{1}\right) \\
& \mathrm{h}=\left(\mathrm{p}_{1}-\mathrm{p}_{2}\right) /\left(\cot \theta_{2} \pm \cot \theta_{1}\right)
\end{aligned}
$$


where $\mathrm{h}$ denotes the height of the scattered point, $\mathrm{p}_{1,2}$ the absolute parallax and $\theta_{1,2}$ the incidence angles in relation with a radar position $0_{1,2}$ (see Fig. 1). The denominator sign depends on the flight configuration: "+" for opposite-side and "-" for same-side flight.

Stereoscopy methods, which are used with optical systems since tens of years, are not applicable directly in the radar systems. Actually, the transposition of the optical methods implies to take into account specificities of the radar images, especially the radiometric situation (speckle noise) and the image creation in slant-range (ground projection in slantrange).

\section{STUDIES ON SIMULATED DATA}

First, we use simulated SAR images (see Fig. 2) from scenes composed of several scatterers: this simple model enables us to validate the first methods and to compare the results with theory [2]. Moreover, this model concentrates on geometrical problems, disregarding radiometric's ones. The developed tool treating the stereoscopic images carries out a simulation which is set up different parameters (viewing angle, sensor altitude and velocity, antenna resolution) of SAR images, a stereo matching between two images acquired from different sensor positions and a 3D stereo intersection to compute elevation. To simplify the model, we consider parallel flights at constant altitude $\mathrm{H}$ and we don't take into account the rotundity of Earth in computations.
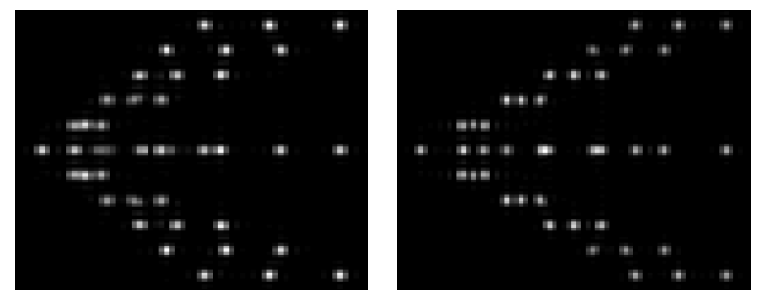

Figure 2. Simulated SAR images

The criterion used to analyze a stereo configuration is the base-to-height ratio $(\mathrm{B} / \mathrm{H}$, see Fig. 1), by analogy with photogrammetry. Two stereoscopic configurations are studied: sensors with a viewing on the same side of the illuminated area (same-side configuration) or on both sides (opposite-side configuration: [5]). Once the altitude extracted by the means of this first radargrammetric tool, we compute the elevation errors between the extracted DEM and the initial 3D scene. The obtained results of the Fig. 3 agree with the theoretical [2] and practical [6] [3] studies on this subject.

The use of scatterers as a radar scene makes possible to concentrate on the geometry of the problem by disregarding radiometric values. It permits us to trust our program because results are those expected. So, we made evolve these algorithms in order to work on real SAR images, taking into account radiometry in matching stage.
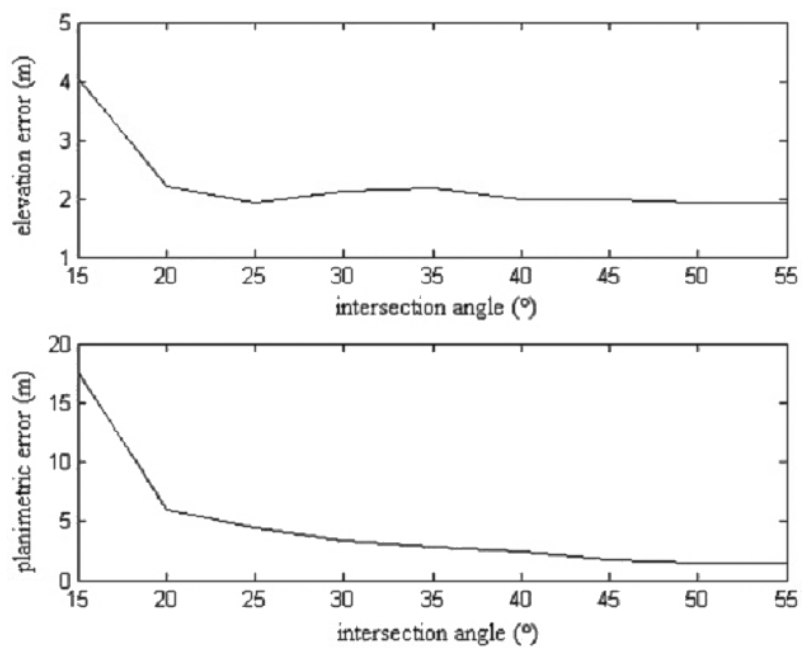

Figure 3. planimetric and elevations errors in same-side configuration

\section{STUDIES ON REAL DATA}

For the matching stage, we prefer to work on a same-side configuration thanks to the radiometric and geometric similarities between the two images. Moreover, it's difficult to deal with image taken from opposite-side configuration because of high radiometric differences and inversed shadows phenomenon.

\section{A. Image informations}

We use stereoscopic images taken from same-side configuration (see Fig. 4). These images focus on the Oetztal area, a rugged terrain in the Austrian Alps [7]. The SIRC/X-SAR mission imaged this area in April 1994. The incidence angles are $58.1^{\circ}$ and $50.3^{\circ}$, the pixel size is $25 \mathrm{~m} \mathrm{x}$ $25 \mathrm{~m}$ and the image size is $568 \times 568$ pixels. The elevation range is $1750 \mathrm{~m}$ to $3750 \mathrm{~m}$.
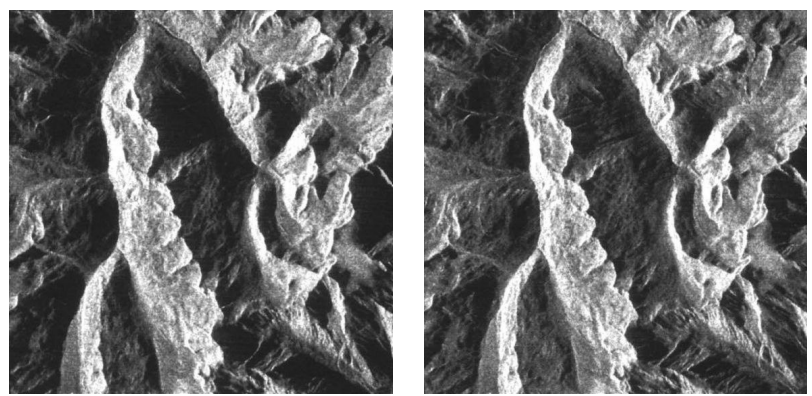

Figure 4. Oetzal site stereo pair $-568 \times 568$ pixels $-25 \mathrm{~m}$ resolution

\section{B. Matching process}

The matching operation between two SAR images is a crucial stage of the processing. It consists in determining for each point in the first image a corresponding point inside the second image, which will be the homologous pixel.

For SAR images, the matching process is often based on the two-dimensional normalized correlation. Another methods exist: edges or regions recognition and feature-based 
techniques. The computation of the normalized crosscorrelation coefficient on gray scale is commonly used on SAR images. In our algorithm, the computation of the correlation coefficients follows (3).

$$
r=\frac{\sum_{m} \sum_{n}\left(A_{m n}-\bar{A}\right)\left(B_{m n}-\bar{B}\right)}{\sqrt{\left(\sum_{m} \sum_{n}\left(A_{m n}-\bar{A}\right)^{2}\right)\left(\sum_{m} \sum_{n}\left(B_{m n}-\bar{B}\right)^{2}\right)}}
$$

where $\mathrm{A}$ is a left image window centered on the pixel to match, $\mathrm{B}$ is a right image window centered on a matching candidate pixel, $\mathrm{m}$ and $\mathrm{n}$ are the range and azimuth size of the windows.

The maximum correlation coefficient permits to indicate which pixels are homologous.

\section{Search area}

The image matching is a time-cost process because (3) is time-consuming and we need to find homologous pixels on a large area.

Considering parallel flights at constant altitude and using epipolar geometry [8], we can reduce the search area assuming that for a given point in an image, the corresponding point in the second image locates on the same azimuth line. Ideally, the search area can be reduced on a thin strip of one pixel thickness on the epipolar line. Practically, it's preferable to have a reduced search area of one to three pixels length in the azimuth direction because estimation errors can lead to mistaken parameters.

The search area can also be limited in the range direction: knowing the minimum and the maximum elevation of the area, we compute the minimum and maximum disparities in range direction using (2).

Finally, the epipolar geometry considerably reduces the size of the search area and reduces computing time. Moreover, it limits false matching because for one pixel to match, there are fewer candidates on the other image.

\section{Pyramidal scheme}

A hierarchical strategy is used to reduce process time and permits to work on large images [9] [10]. The principle is quite simple: from the original image, we build an image pyramid shown in Fig. 5. At each level, the image size is reduced by a factor $2^{\mathrm{k}}$ corresponding to the k-iteration step. The images are reduced by averaging the pixels gray levels: in the reduced image, each pixel value is the average of four pixels in the previous image. For each iteration, the matching process permits to establish an approximate disparity map. Thus, we are able to predict the disparity offsets at the next level of the hierarchical process, reducing computation time and speckle errors. With increasing iterations, we obtain a better accuracy for each level. At the final step, the last disparity map is used to produce the DEM.

\section{E. Sub-pixel accuracy}

With $25 \mathrm{~m}$ resolution images, a one-pixel disparity corresponds to a $25 \mathrm{~m}$ distance disparity. So, (2) leads to an elevation accuracy equals to $120 \mathrm{~m}$.

If we want to improve this accuracy we need to obtain a sub-pixel accuracy for the disparity map. That is possible if we interpolate original images in order to enlarge them by a factor $2^{\mathrm{k}}$. The method used is the bilinear interpolation, for $\mathrm{k}=1$ and $k=2$. Theoretically, the disparity and accuracy are also reduced by the factor $2^{\mathrm{k}}$, which leads to an elevation accuracy equals to $30 \mathrm{~m}$.

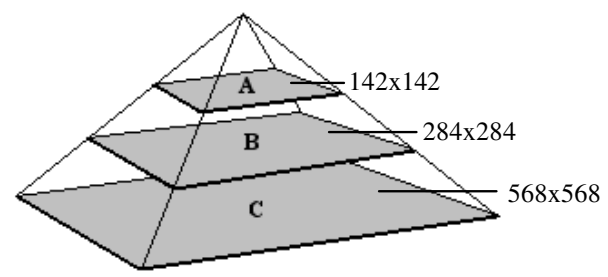

Figure 5. Pyramidal scheme with growing resolutions

These larger images are added at the end of the pyramidal scheme to complete the hierarchical process.

\section{F. Results}

Using all the methods mentioned before, we obtain the DEM shown in Fig. 6. The elevation values and global appearance agree with the reference DEM [7] presented on Fig. 7. However, there are lots of errors in the low radiometric levels areas. Indeed, in such areas, it's difficult to discriminate pixels and there are a lot of mismatched points. These errors involve disparity and elevation errors.

To avoid these errors, we detect homogeneous low radiometric level areas to enlarge correlation window in this case. We obtain some improvements and the corrected DEM (see Fig. 8) is quite similar to the reference DEM (Fig. 9).

Comparing the DEM we obtained with the reference DEM, we obtain the results presented in Tab. 1 . We notice that the elevation errors between the two DEM are generally from $10 \mathrm{~m}$ to $130 \mathrm{~m}$, excluding shadowed areas. The relative elevation errors are from $0.3 \%$ to $5.8 \%$.

TABLE I. ELEVATION ERRORS ON THE GENERATED DEM

\begin{tabular}{|c|c|c|c|}
\hline Elevation errors & 0 to $70 \mathrm{~m}$ & 70 to $130 \mathrm{~m}$ & 130 to $200 \mathrm{~m}$ \\
\hline $\begin{array}{l}\text { Percentage of pixels on the } \\
\text { concerned error range }\end{array}$ & $45 \%$ & $40 \%$ & $15 \%$ \\
\hline Relative elevation errors & 0 to $2 \%$ & 2 to $4 \%$ & 4 to $6 \%$ \\
\hline $\begin{array}{l}\text { Percentage of pixels on the } \\
\text { concerned error range }\end{array}$ & $25 \%$ & $55 \%$ & $20 \%$ \\
\hline
\end{tabular}



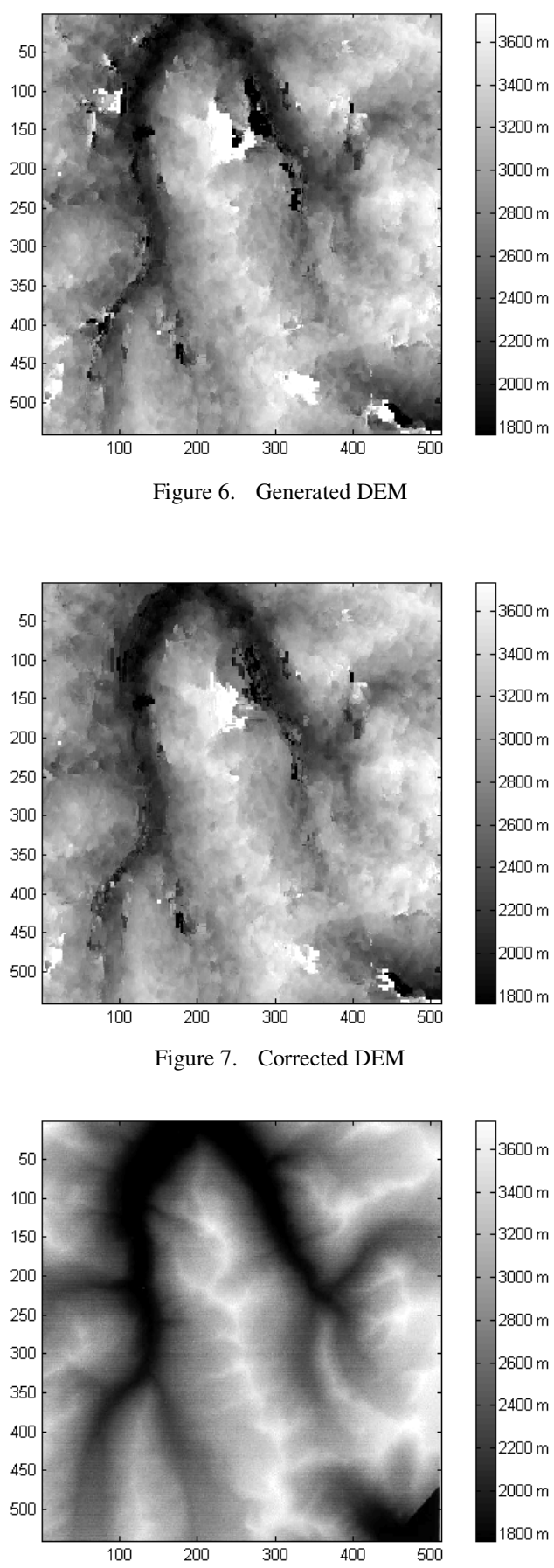

Figure 8. Reference DEM
Several mistakes are corrected but others still remain especially in the mentioned areas. So it will be necessary to improve our method to deal with low radiometric level areas.

\section{CONCLUSION AND FURTHER WORKS}

Using a simple spatio-triangulation model enabled us to validate the computation of our first radargrammetric tool. The results are in agreement with the theoretical studies so that we made evolve our program to work on real SAR images.

To have a correct matching stage, we focus on the use of a pyramidal algorithm and on the discrimination of the homologous pixels. One of the next evolutions of this tool is to correct DEM in homogeneous areas such a low radiometric level areas. So, the use of polarimetric information should extract the mentioned areas in order to apply a particular processing.

The encouraging results incite us to work on a mountainous site on which we have DEM data and two SIRC images recorded with $35^{\circ}$ and $50^{\circ}$ incidence angles. The main problem is such an angle difference involves more dissimilarity between images. Moreover, high relief areas are not very collaborative for the matching process because of larger disparities. The first results show that appropriated treatments are needed to deal with these new geometric parameters.

\section{REFERENCES}

[1] F. Fayard, S. Méric, and E. Pottier, "First studies on a radargrammetric tool kit," EUSAR Conference, 2006.

[2] F. Leberl, "Radargrammetric image processing," Artech House, 1990.

[3] T. Toutin, and L. Gray, "State-of-the-art of elevation extraction from satellite SAR data," ISPRS Journal of Photogrammetry \& Remote Sensing, Vol 55, 2000, pp. 13-33.

[4] Y. Belgued, L. Rognant, L. Denise, S. Goze, and J.G. Planés, "Application of radar space triangulation to the calibration of interferometric DEM," IGARSS'98, IEEE international published, Vol.5, 1998, pp. 2665-2667.

[5] J.K. Fullerton, F. Leberl, and R.E. Marque, "Opposite-side SAR image processing for stereo-viewing," Photogrammetric Engineering and Remote Sensing, Vol. 52, No. 9, 1986, pp. 1487-1498.

[6] Y. Belgued, L. Denise, S. Goze, and J.G. Planés, "Dimensionnement des performances de la localisation spatiale radar," Bulletin SFPT $\mathrm{n}^{\circ} 155$, 1999.

[7] P. Paillou, and M. Gelautz "Relief reconstruction from SAR stereo pairs: the optimal gradient matching method," IEEE Transactions on Geoscience and Remote Sensing, Vol 37, N 4, July 1999, pp. 20992107.

[8] Z. Zhang, R. Deriche, O. Faugeras, and Q.T. Luong, "A robust technique for matching two uncalibrated images through the recovery of the unknown epipolar geometry," Artificial Intelligence, Vol. 78, 1995, pp. 87-119.

[9] M. Denos, "A pyramidal scheme for stereo matching SIR-B imagery," Int. J. Remote Sensing, Vol. 13, No. 2, 1992, pp. 387-392.

[10] H. Maître, "Traitement des images de RSO," Hermès Science Publications, 2001. 